\title{
Robot assisted laparoscopic excision of a paraganglioma: new therapeutic approach
}

\author{
G. Cochetti, E. Cottini, F. Barillaro, E. Lepri, A. Boni, S. Pohja, E. Mearini \\ Department of surgical specialties and Public Health (DZM), Urological Andrological Surgery and \\ Minimally Invasive Techniques (CG, BF, BA, ME), University of Perugia, Perugia-Terni, Italy
}

\begin{abstract}
The Paraganglioma is the most common extra-adrenal pheochromocytoma arising from neural crest (1) (It will better to write: The paraganglioma is an extra-adrenal pheocromocytoma arising from the neural crest. 10\% of pheocromocytomas are extra-adrenal and can arise form chromaffin tissue derived from primitive neuroectoderm). Minimally invasive techniques allow surgeons to perform the procedure without wide exposure and mobilization of intra abdominal organs. To our knowledge we present the third case of robotic excision of a retroperitoneal paraganglioma $(2,3)$.
\end{abstract}

\section{MATERIAL AND METHODS}

A 31 years old male with incidental $3 \times 3$ $\mathrm{cm}$ retroperitoneal mass located between the left renal vein and aorta. Even if serum Neuron-Specific Enolase and chromogranine-A were negative an anti-hypertensive prophylaxis was administered. (Will be worthwhile to add if cathecolamines E.g. metanephrines where measured in urine as part of the most common lab tests used when pheocromocytoma is suspected. Also if possible the type of anti-hypertensive drug used). We preferred a retroperitoneal approach. The ureter and the spermatic vessels were identified and not resected. The peritoneum was incised at the level of the tumor and the mass was carefully dissected. The maneu- ver was difficult because the paraganglioma was characterized by many small vessels leading to the lesion, unlike adrenal pheochromocytoma where a definite pedicle may be identified. Robotic surgery allowed to identify the vascular branches and to clip them with $5 \mathrm{~mm}$ Hem-o-Lok. The manipulation of the mass caused some hypertensive peaks up to $240 / 130 \mathrm{mmHg}$. For this reason, we were forced to perform a step by step dissection. Finally, the tumor was inserted into an EndoBag and retrieved from the optical trocar.

\section{RESULTS}

Operative time was 120 minutes; estimated blood loss was less than 50cc. No complications occurred. After surgery the blood pressure was normal. Patient was discharged on postoperative day 2. Final histopathological exam showed paraganglioma (a short pathology description would be helpful to add a little more information to the video case report).

\section{CONCLUSIONS}

The robotic excision of paraganglioma is a safe (a comment on the extremely high blood pressure during surgery should be added in order to show that this could be a high risk surgery when precautions prior to surgery are not made) and efficacious surgical strategy. 


\section{REFERENCES}

1. Boneschi M, Erba M, Giuffrida GF, Mattioli A, Miani S: Functioning paraganglioma. Minerva Chir. 1993; 48: 1399-405.

2. Park JS, Lee KY, Kim JK, Yoon DS: The first laparoscopic resection of extra-adrenal pheochromocytoma using the da Vinci robotic system. J Laparoendosc Adv Surg Tech A. 2009; 19: $63-5$.

3. Lehrfeld T, Natale R, Sharma S, Mendoza PJ, Schwab li CW, Lee DI: Robot-assisted excision of a retroperitoneal mass between the left renal artery and vein. JSLS. 2010; 14: 447-9.
Correspondence address:

Cochetti Giovanni, MD

Department of surgical specialties and Public Health Urological Andrological Surgery and Minimally Invasive Techniques University of Perugia, Perugia-Terni, Italy P.zza Università 1 Perugia, 06123, Italy E-mail: giovannicochetti@libero.it

\section{ARTICLE INFO}

Available at: www.brazjurol.com.br/videos/march_april_2014/Giovanni_279_280video.htm

Int Braz J Urol. 2014; 40 (Video \#4): 279-80

Submitted for publication: December 15, 2013

Accepted after revision: February 26, 2014

\section{EDITORIAL COMMENT}

In the video by Dr. Cochetti and colleagues, a robotic assisted laparoscopic excision of a paraganglioma is well illustrated constituting the third such reported case in the scientific literature.

Paragangliomas are not common, they represent only $10 \%$ of the pheocromocytomas in adults. The diagnosis requires a high index of clinical suspicion. In this context, a very thorough history taking and physical examination to look for signs and symptoms of catecholamine excessive release in those patients with functional lesions is pivotal. This will be critical as the patient will need pre-operative antihypertension management in order to avoid potential elevations of blood presure and tachycardia leading to potential catastrophic hemodynamic sequelae in the peri-operative period.
As with other surgical procedures, minimally invasive techniques offer not only cosmetic advantages but also a quicker recovery and less surgicery related pain. In the case of paragangliomas, minimally invasive surgery will add improved optics and 3-dimensional visualization of the incipient lesion, with surgical magnification of important anatomical structures including feeding (arterial and venous) vessels whereby avoiding excesive manipulation of the paraganglioma that can have far reaching implications pertaining to patient outcome. 